\title{
Directed-assembly of carbon structures in a nonpolar dielectric liquid under the influence of DC-generated electric fields
}

Luciana Oliveira ${ }^{1}$, Deepika Saini ${ }^{2,3}$, Jay B. Gaillard ${ }^{3}$, Ramakrishna Podila ${ }^{2}$, Apparao M. Rao ${ }^{2 *}$, and Steven M. Serkiz ${ }^{1,2,3^{*}}$

1. School of Materials Science and Engineering, Clemson University, Clemson, SC USA 29634

2. Department of Physics and Astronomy, Clemson Nanomaterials Center, and COMSET, Clemson University, Clemson, SC USA 29634

3. Savannah River National Laboratory, Aiken, SC USA 29808

\begin{abstract}
Externally applied direct current (DC) electric fields have been examined as a means of controllable organization of carbon structures (one-dimensional multi-walled carbon nanotubes of three sizes, quasi two-dimensional exfoliated graphene platelets, and three-dimensional bulk graphite) suspended in a high dielectric strength (i.e., highly resistive) solvent (perfluorocarbon FC-40). The net particle charge of the carbon structures in FC-40 was negligible. This eliminates non-dielectrophoretic (DEP) kinetic motions and allows for examination of isolated DEP forces on the assembly process. At a sufficiently high DC field strength and carbon structure concentration, DEP directed assembly and subsequent formation of electrically conductive networks were observed. The influences of particle size, aspect ratio, concentration, and structure on the assembly and electrical conduction in this system were investigated. Threshold voltage, an operationally defined measure of the applied voltage where current flow first occurred, was used as a characteristic measure of the assembly process. Consistent with charge percolation theory, the threshold voltage was found to be inversely related to the particle concentration and directly proportional to particle size as measured by light scattering. Accordingly, we developed an agglomeration model that accounts for the influence of particle size on the threshold voltage.
\end{abstract}

\footnotetext{
* Corresponding author. Email: steven.serkiz@srnl.doe.gov (Steven M. Serkiz)

Email: arao@clemson.edu (Apparao M. Rao)
} 


\section{INTRODUCTION}

Self-assembly plays a vital role in nature and is often found in a wide range of physical[1], chemical and biological systems[2,3]. Drawing on the behavior of these systems, researchers have been investigating self or directed organization processes in order to design complex devices with tailored capabilities such as selective sensing[4], optoelectronics[5], controlled photonic bandgap[6,7], etc. The discovery of low-dimensional materials such as the carbon nanotube and graphene nanoplatelets have provided the much-needed breakthroughs in these research efforts owing to their exceptional electrical, mechanical and thermal behavior. In order to harness these promising properties, a concerted effort has been put forth in building organized networks for fabrication of thin film transistors[8, 9], conducting coatings[10], and electromechanical devices such as flexible electronics[11, 12] and moldable integrated circuits[13], to name a few. Researchers have developed a variety of directed-assembly approaches based on intermolecular forces[14], external fields [15, 16], and viscous channels[17]. Of particular interest to this article is the electric field-guided dielectrophoretic (DEP) assembly process which is a viable route to realize a high throughput fabrication method. In this technique, a non-uniform external electric field $(E)$ induces a dipole moment $(p)$ in suspended particles, as a result of which the particles experience a DEP force, $\vec{F}_{D E P}=(\vec{p} \bullet \nabla) \vec{E}$ and a torque, $\tau=\vec{p} \times \vec{E}$. Under the conditions of non-uniform $E$, this force can reorient and propel particles towards (positive DEP) or away from (negative DEP) the maximum electric strength. Considering that the induced dipole moment is material-property dependent, particle response to an applied field is largely determined by its physicochemical properties (composition, type, size, alignment, and aspect ratio[18]). Under certain conditions of applied potential and particle concentration, the suspension can undergo an assembly process to form an interconnected network of particles, resulting in electrically conducting percolation paths. These seemingly random particle assembly dynamics can be described by a percolation model, an extensive mathematical theory fundamentally based on probability. It is noteworthy that though both $\mathrm{AC}$ and DC fields can trigger the assembly, a simple DC field is not often the preferred choice, as it suffers from complicating effects such as the electrophoresis of solvent molecules, potential electrochemical reactions and non-DEP induced electrokinetic movement of suspended 
particles[19, 20]. Physical and chemical properties of the particles, as well as the solvent, have a profound impact on the development of percolation pathways. Owing to the complexity of the self-assembly dynamics, the effect of these physicochemical properties have yet to be completely understood.

In this study, we investigated the development of electrically conducting networks of carbon structures viz. quasi 1D multi-walled carbon nanotubes (of three different sizes), 2D graphene platelets and 3D bulk graphite in non-polar FC-40, a high dielectric strength $\left(\sim 18 \times 10^{6} \mathrm{~V} / \mathrm{m}\right)$ and high electrical resistivity $\left(4.0 \times 10^{13} \Omega \bullet \mathrm{m}\right)$, solvent. In this solvent, the suspended carbon structures were found to be uncharged which eliminated any electrokinetics other than the dielectrophoresis and allowed us to apply $E$ (DC voltages) between $10 \mathrm{kV} / \mathrm{m}-1000 \mathrm{kV} / \mathrm{m}(0.1-10$ $\mathrm{kV}$ ). This study elucidates the influences of material physicochemical properties and field strength on the directed-assembly process and subsequent formation of charge percolation networks.

\section{EXPERIMENTAL}

In order to characterize the directed-assembly behavior of carbon structures under a DEP force, we investigated suspensions of randomly oriented multi-walled carbon nanotubes (R-MWCNTs), long vertically aligned multi-walled carbon nanotubes (VA-MWCNTs) of $100 \mu \mathrm{m}$ and $1 \mathrm{~mm}$ length, mechanically exfoliated graphene platelets (EGPs), and bulk graphite powders in FC-40.

The materials were acquired as follows: R-MWCNTs (diameter: 20-30 nm) from Cheap Tubes, Inc (Brattleboro, VT USA), $100 \mu \mathrm{m}$ and $1 \mathrm{~mm}$ long VA-MWCNTs (diameter: 20-25 $\mathrm{nm}$ ) synthesized using the ferrocene/xylene based chemical vapor deposition method[21]. Three different kinds of MWCNTs were employed to investigate the effects of nanotube dimensions on the assembly behavior. Grade $M$ graphene nanoplatelets (GPs) with an average thickness of approximately 6-8 $\mathrm{nm}$ and a width of $5 \mu \mathrm{m}$ were purchased from XG Sciences, Inc. (Lansing, MI USA). The as-purchased GPs were exfoliated in N-Methylpyrrolidone (NMP) for 2 hours using a tip sonicator, sonicated a second time in a fresh portion of NMP for an additional 6 hours, and separated by vacuum filtration using a $0.45 \mu \mathrm{m}$ nylon membrane filter. The exfoliated GPs (EGPs) were dried in an oven at $130{ }^{0} \mathrm{C}$ for 24 hours to obtain the final sample used in 
suspension preparations. Bulk graphite powder (crystalline, 300 mesh, 99\%) from Alfa-Aeser was used as received.

A high dielectric perfluorocarbon (FC-40, from $3 \mathrm{M}^{\mathrm{TM}}$ ) was used as-received. This solvent was selected for this study for its high resistivity and high dielectric strength allowing us to study the particle response under high electric fields.

The carbon structures were suspended in FC-40 at predetermined concentrations $(1 \mu \mathrm{g} / \mathrm{mL}$ to $1000 \mu \mathrm{g} / \mathrm{mL}$ ) and sonicated for $~ 5$ min using a 1/8" tip sonicator (Branson-250) to homogenize the suspension. The effective charge on the particles was determined by Zeta potential measurement (Brookhaven nano90+ particle analyzer).

Material Characterization: All carbon structures were characterized in dry form using scanning electron microscopy (SEM Hitachi S4800), Fig 1, and in the FC-40 solvent with dynamic light scattering (Brookhaven nano90+ analyzer). The reported particle sizes were measured at the percolation threshold concentration for each of the carbon structure suspensions (described in the later sections). Suspensions of the corresponding threshold concentration were then drop casted and dried for imaging under the SEM. 


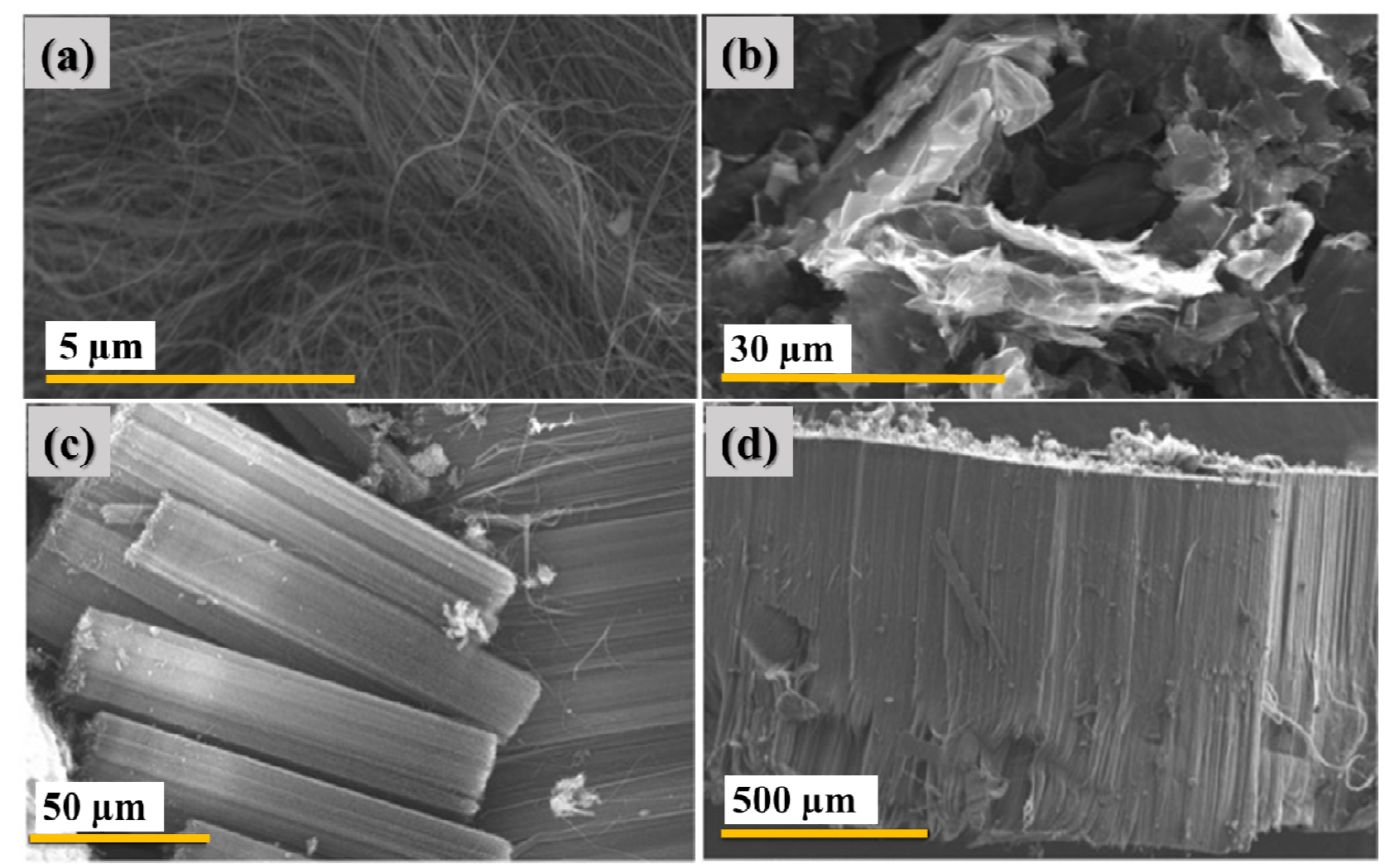

Figure 1. Scanning electron micrographs for: a) R-MWCNTs which show the lack of a preferred orientation of MWCNTs; b) EGPs obtained after sonication in NMP for 8 hours. The average surface area of graphene flakes is in the range of $25-50 \mu \mathrm{m}^{2}$ and 5-10 layers in thickness; c) and d) $100 \mu \mathrm{m}$ and $1 \mathrm{~mm}$ long VA-MWCNTs in which the MWCNTS are oriented. For maintaining a consistency, the images were taken at the respective concentration threshold (minimum particle concentration required for current flow to occur at less than $10 \mathrm{kV}$, described in later sections).

Directed-assembly studies: The DC field-induced assembly of carbon structures in FC-40 was examined in 3D using a cylindrical glass vial (radius $\sim 1 \mathrm{~cm}$, height $\sim 3 \mathrm{~cm}$ ) with $2 \mathrm{~cm}$ long and $0.8 \mathrm{~mm}$ thick paperclips as the electrodes (see Fig. 2a). The sonicated suspensions were poured into the vial and the potential applied across the electrodes at a constant ramp rate $(\sim 0.6 \mathrm{~V} / \mathrm{s})$ using a high voltage supply (current limit: $400 \mu \mathrm{A}$ ), schematic shown in Fig. 2b. The applied 
potential and current across the electrodes were simultaneously recorded as a function of time using serial port RS 232 computer interface.

(a)

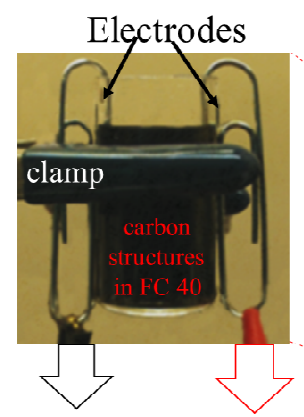

To power supply (b)

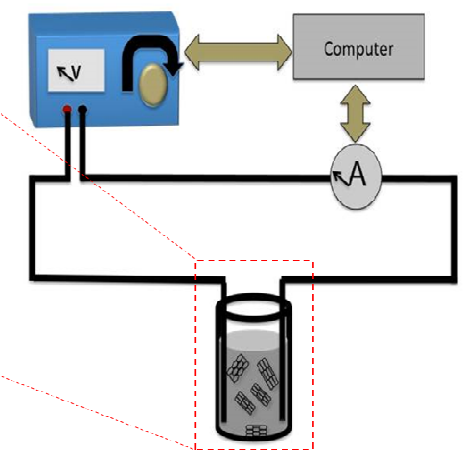

(c)

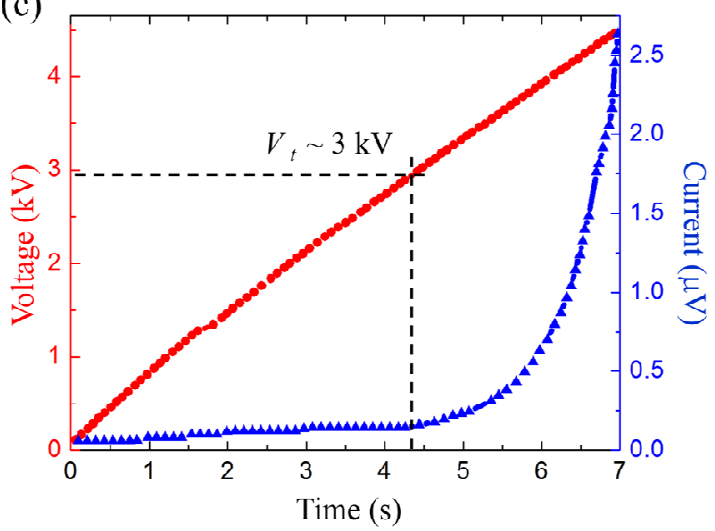

Figure 2. a) Photograph of experimental set up of a test cell (note: vial diameter $\approx 1 \mathrm{~cm}$ ). b)

The schematic shows the cell and electrode geometry used for studying the self-assembly of carbon structure suspensions in FC-40. An increasing voltage $V$ is applied across the electrodes at a constant ramp rate (red trace in (c)) and the corresponding current $I$ (blue trace in (c)) is measured using a computer controlled ammeter c) typical $I$ - $V$ curve shown is for a $50 \mu \mathrm{g} / \mathrm{mL}$ RMWCNTs suspension in FC-40, exhibiting an exponential increase in the current beyond the threshold voltage as defined as the inflection point (indicated by the vertical dashed line).

Real-time imaging: For a selected subset of the carbon MWCNTs suspensions, directed assembly process in a quasi 2D geometry (i.e., thin film) was imaged in real time using 10x objective (15 mm work distance) of Nikon Epithot Inverted Dark Field microscope coupled to a Nikon Sight DS Fil camera (see supplementary information). Larger diameter (70 nm-80 nm; M- grade MWCNTs from Nanotech Labs) were used for higher visibility under the dark field microscope.

COMSOL simulations: The electric-field distribution for the setup shown in Fig. 2 was modeled using COMSOL ${ }^{\circledR}$ multiphysics software (COMSOL Inc., Los Angeles, CA). For simulation 
purposes, the dielectric constant for FC-40 medium was set to 1, and the dimensions of the electrodes were those of the experimental set up.

\section{RESULTS AND DISCUSSION}

As shown in Fig. 2c, the typical current $(I)$ versus voltage $(V)$ curves for the carbon structure suspensions in FC-40 exhibit an exponential increase in $I$ beyond a threshold voltage $\left(V_{t}\right)$. This operationally defined measure allows for a simple comparison of I-V curves from different experiments and is defined as the inflection point of the exponential curve (indicated by the dashed lines in Fig. 2c) resulting from the directed assembly. In other words, the I-V data exhibit a highly nonlinear and time dependent $\mathrm{d} I / \mathrm{d} V$ (or conductance). The directed-assembly does not occur below $V_{t}$ indicating that the particles remain stationary until strong enough field gradients are generated to initiate the attraction of the suspended particles near the electrodes.

The formation of a conducting path is sensitive to particle-size distribution in the suspension and this distribution is influenced by the degree of agglomeration of carbon structures. Hence, experiments at each concentration were conducted in triplicate and the $V_{t}$ values reported are the average of these three results. 
(a)

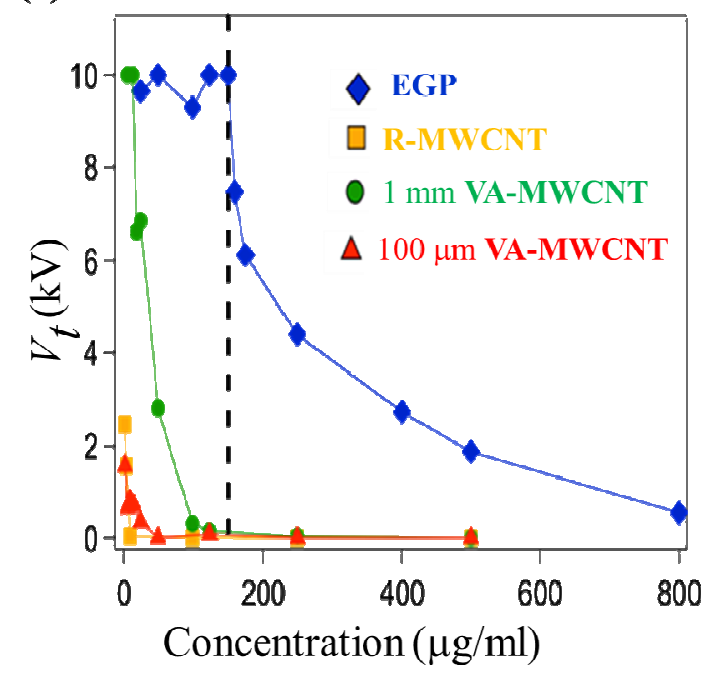

(b)

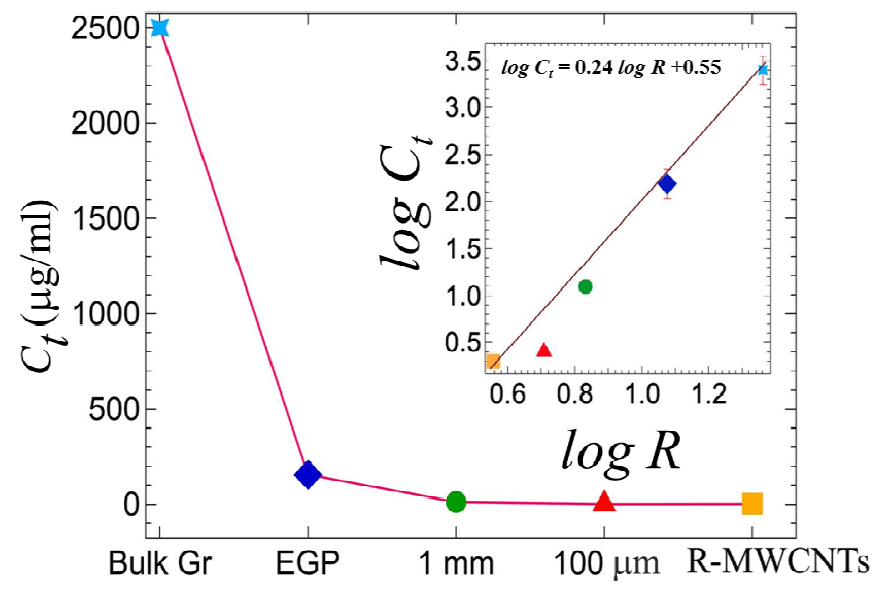

Figure 3. a) The threshold voltage, $V_{t}$ plotted as a function of carbon structure concentration in FC-40. The dashed vertical line indicates a threshold concentration, $C_{t}$, of $155 \mu \mathrm{g} / \mathrm{mL}$ for a dispersion of EGPs. b) The variation of $C_{t}$ for different carbon based materials. The inset shows a logarithmic plot of $C_{t}$ as a function of measured hydrodynamic particle size $(R)$ of the carbon structures in FC-40.

As predicted by the percolation theory, $V_{t}$ decreases with increasing MWCNT/EGP concentrations (Fig. 3a). Such a result is expected because an increase in particle concentration results in an increase in the junction density (number of connections per unit volume) between the particles.

A directed-assembly threshold concentration $\left(C_{t}\right)$ was defined as the minimum concentration at which carbon structures form an electrically conducting path with an applied voltage between 0 and $10 \mathrm{kV}$ (Fig. 3a), and was measured at a constant applied potential ramp rate of $\sim 0.5-1$ $\mathrm{kV} / \mathrm{sec}$. For example, the dashed line in Fig. 3a shows that $C_{t}$ for EGPs is $\sim 155 \mu \mathrm{g} / \mathrm{mL}$, (i.e., below $155 \mu \mathrm{g} / \mathrm{mL}$ a voltage $>10 \mathrm{kV}$ must applied to initiate directed-assembly). For these experiments, we used an upper limit of $10 \mathrm{kV}$ to avoid any sparking between the electrodes. We observed that the $C_{t}$ values, Fig. $3 \mathrm{~b}$ and Table 1, were different for various types carbon structures evaluated. Interestingly, $C_{t}$ directly relates to the measured hydrodynamic particle size $(R)$ (see inset Fig 3b). 
Real-time imaging of a quasi-2D directed-assembly of MWCNT (Fig. 4 and supplementary information) clearly shows the organization of MWCNTs under the influence of an electric field. As seen from the snapshots of the recorded assembly process, Fig. 4a, MWCNTs are directed to areas of high electric field gradient. Previously, Liu et al. [22] showed that in a quasi 2D geometry (electrodes attached to a flat conducting slab), nanowire (NWs) directed assembly (or the "pearl-chain" formation) occurs via the following four steps: i) DC voltage creates a DEP force and some NWs in the vicinity of the electrodes are attracted towards the electrode tip due to the presence of high field gradient, ii) subsequently, a field gradient is generated near the free end of NWs that are attached to the electrodes, iii) this new field gradient (generated in step ii) leads to a local bundling of NWs, and iv) finally, these local bundles link together to form an electrically conducting path between the electrodes. This assembly mechanism is consistent with our conceptual model, where carbon structures in suspension move towards the interface between the assembled bundles and the bulk suspension. Over time this effect is translated through the gap between the two electrodes to form an electrically conducting network.

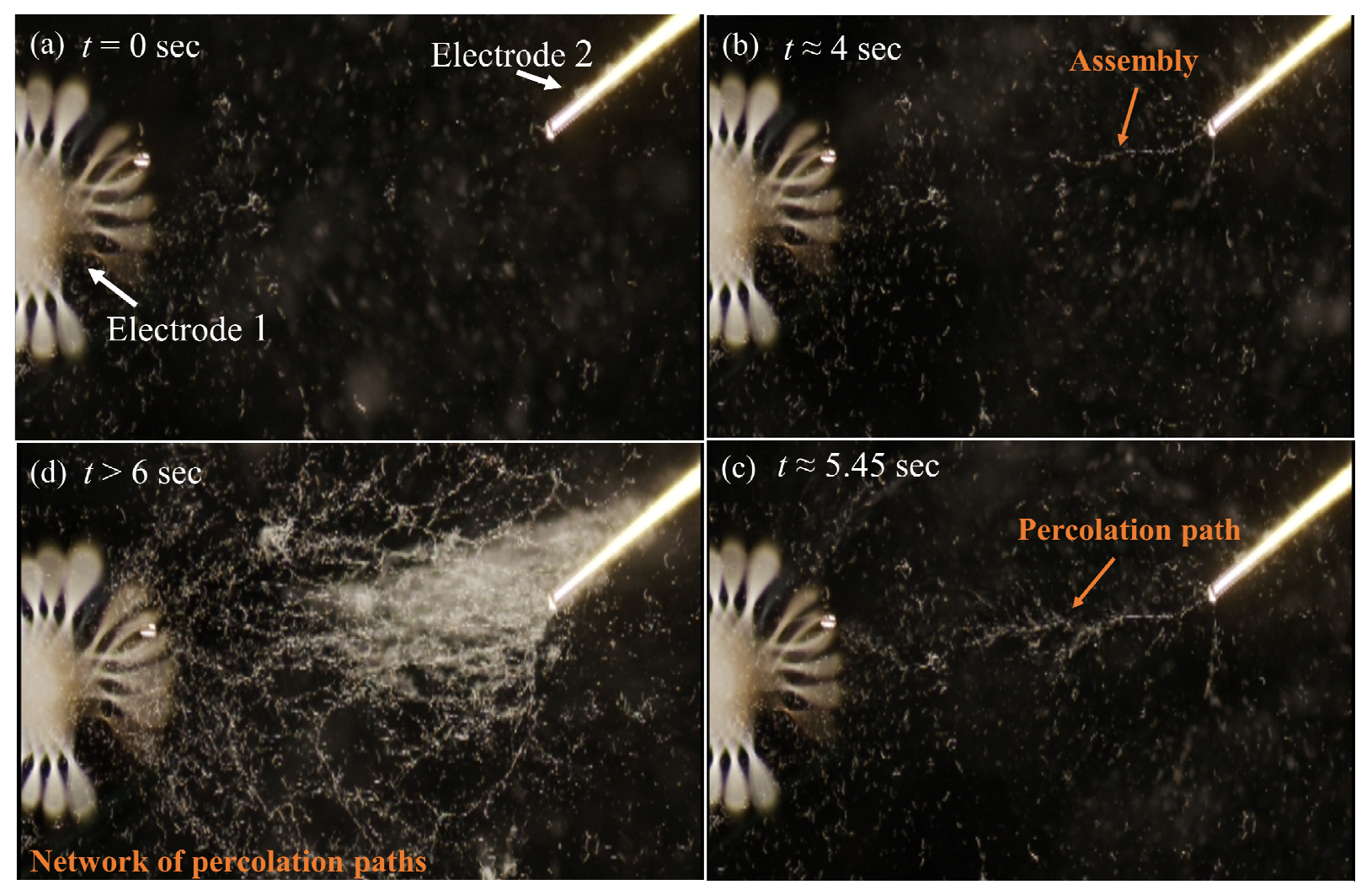


Figure 4: Snapshots from the real-time video of quasi 2D directed-assembly of MWCNTs suspended in FC-40 with a constant applied voltage (through $30 \mu \mathrm{m}$ wire electrodes) of $V \sim 125$

V. a) Suspended MWCNTs can be seen as random elongated "dots". Flower-like image of electrode 1 is due to its orientation and angle of the incident light. b) MWCNTs in close proximity of the electrode align and travel towards the electrodes. The increased electric field gradient created by the aligned MWCNTs connected to the electrode increases the DEP forces and further aligns the neighboring MWCNTs. c) Ultimately a channel of connected MWCNTs grows across the electrodes forming a conducting percolation path. d) Many new percolation paths are formed.

For further investigation of this conceptual model, we simulated the electric-field distribution in our set up ( $c f$. Fig. 1a) using $\mathrm{COMSOL}^{\circledR}$ and predicted the electric-field gradients resulting from an external DC voltage. As shown in Fig. 5, our simulations showed the presence of smooth electric field gradients (x-component of electric field; $E_{x}$, is observed in Fig. 5) in the vicinity of the electrodes (Fig. 5b and 5c). However, these gradients become stronger when suspended particles, say MWCNT as in current simulation, are present in close proximity of the electrode. The model predicts that the MWCNT "decorated" electrode display a much higher nonuniformity in the local electric field (Fig. 5d). $E_{x}$ at the free end of the MWCNT create high field strength hot spots (Fig. 5e). Neighboring particles will thus be attracted to this area and further extend the network until eventually a conducting path of connected MWCNTs will grow between the electrodes, allowing the charge to percolate. Thus the formation of percolation paths predicted by the simulated electric fields corroborates the observed experimental behavior of the carbon structures through the images obtained for MWCNTs (Fig 4). 


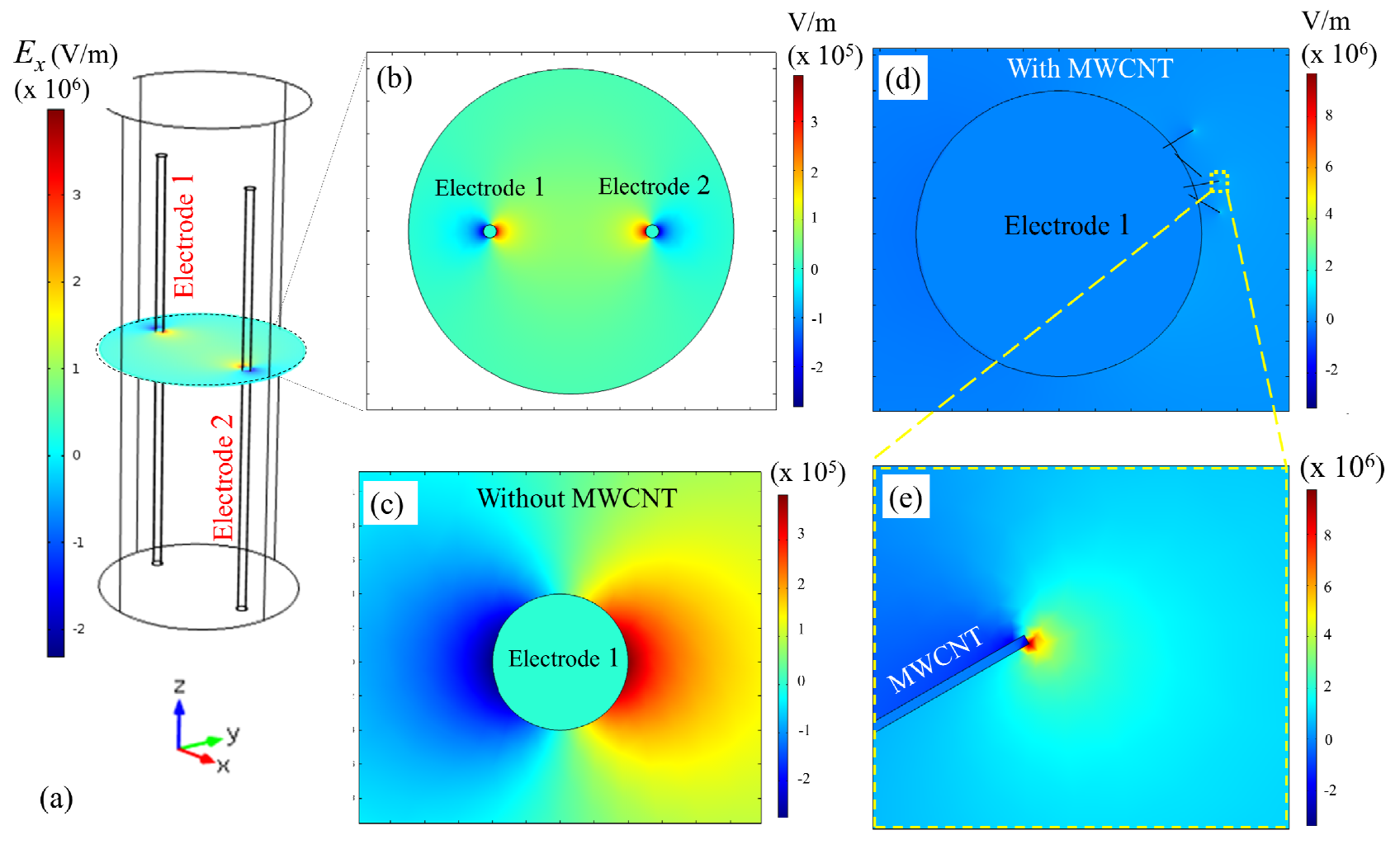

Figure 5: $\mathrm{COMSOL}^{\circledR}$ simulations showing the electric field distribution in our setup. a) 3D set up of the electrodes similar to the experimental apparatus. The electrodes $(0.8 \mathrm{~mm}$ thick $)$ are $3 \mathrm{~cm}$ apart b) 2D map of the x-component electric field $\left(E_{x}\right)$ in the cross section of the set up showing a smooth $E_{\mathrm{x}}$ gradient around the electrodes (c) Enlarged image of electrode 1 in the absence of any MWCNTs (d) A MWCNT decorated electrode shows a quiet different spatial distribution of $E_{x}$. e) High intensity $E_{x}$ hot-spots can be seen at the free edges of MWCNTs. This non-uniformity in the $E_{x}$ enhanced in the presence of the MWCNTs drives the assembly process.

Modeling parameters: The applied DC voltage is $1 \mathrm{kV}$. For computational simplicity, 2D MWCNTs are modeled as $100 \mathrm{~nm}$ x $100 \mu \mathrm{m}$ rectangles. The electrode in the current set up has been modeled with 4 MWCNTs. 
The insights from the computational model and experimental observations allow us to rationalize the observed differences in $C_{t}$ for various carbon structures used in this study, for which a simple model is proposed as follows. Considering the particles to be spherical, the particle number density $\left(n_{p}\right)$ at a given sample concentration $X \mathrm{mg} / \mathrm{mL}$ is given by:

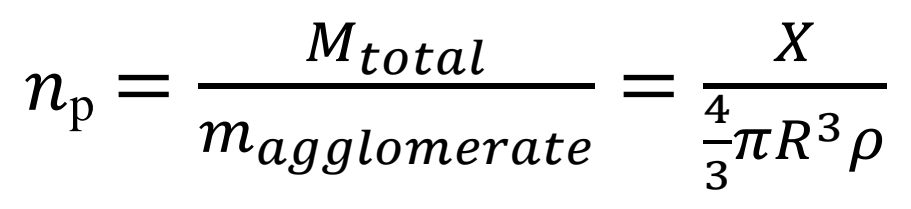

where $M_{\text {total }}$ is the total mass of carbon materials present in the dispersion and $m_{\text {agglomerate }}$ is the mass of the carbon structure agglomerate, $R$ is the hydrodynamic particle size discussed in Fig. $3 \mathrm{~b}$ and $\rho$ is the density of carbon $\left(\sim 2.3 \mathrm{~g} / \mathrm{cm}^{3}\right)[23]$. We know through visual observation and optical microscopy (see assembly video in the supplementary information) that the non-uniform electric field results in an increasing particle concentration in the vicinity of the electrodes. For simplicity however, this effect is ignored in the current model. The model is further simplified by the assumption implicit in Eq. 1 that the particles in suspension are monodispersed. It is important to note that $n_{p}$ varies with hydrodynamic particle size and not the absolute dimensions of the dry carbon structures (i.e., at a given concentration, smaller sized carbon structures agglomerates (low $R$ ) will exhibit higher $n_{p}$ values). $C_{t}$, hence, is inversely proportional to $n_{p}$ because the conducting path is observed to be formed at relatively lower concentrations for particles with a smaller $R$ (i.e. increased $n_{p}$ ), Table 1 . The absence of any dramatic difference between $C_{t}$ of $100 \mu \mathrm{m}$ and $1 \mathrm{~mm}$ MWCNTs is consistent with the model based on agglomerate size rather than dry particle size. Considering $C_{t} \propto A R^{\beta}$, where the exponent $\beta$ is the dimensionality constant (dependent on hydrodynamic shape) of carbon structures and $A$ is a proportionality constant determined by fitting the model described by Eq. 2 .

$$
\log C_{t}=\beta \log R+A
$$

For spherical particles, $C_{t}$ should be directly proportional to $R^{3}$ i.e. $\beta$ is expected to be $\sim 3$. When Eq. 2 was fitted to the $C_{t}$ values for various carbon structures (obtained from Fig. 3a), $\beta \sim 0.24$ (see inset in Fig. 3b) was the best fit. This much lower $\beta$ value can be attributed to the high aspect ratio of the particles under study which possibly results in non-spherical agglomerates. 


\begin{tabular}{|l|l|l|l|l|l|}
\hline Carbon Structures & $\begin{array}{l}\text { Bulk } \\
\text { graphite } \\
\text { powder }\end{array}$ & EGPs & $\begin{array}{l}\text { VA-MWCNTs } \\
1 \mathrm{~mm}\end{array}$ & $\begin{array}{l}\text { VA-MWCNTs } \\
100 \mu \mathrm{m}\end{array}$ & R-MWCNTs \\
\hline $\begin{array}{l}\text { Hydrodynamic } \\
\text { size; } R \\
(\mu \mathrm{m})\end{array}$ & $23.0 \pm 5.7$ & $\begin{array}{l}11.9 \quad \pm \\
6\end{array}$ & $6.8 \pm 1.7$ & $5.1 \pm 2.3$ & $3.6 \pm 0.6$ \\
\hline $\begin{array}{l}\text { Threshold } \\
\begin{array}{l}\text { Concentration; } C_{t} \\
(\mu \mathrm{g} / \mathrm{ml})\end{array}\end{array}$ & 2500 & 155 & 19 & 2.5 & 2 \\
\hline
\end{tabular}

Table 1: The hydrodynamic size (inferred from dynamic light scattering measurements) and corresponding threshold concentrations of the carbon structures, when suspended in FC-40.

It is worth noting that although the percolation threshold concentration appears to be function of the hydrodynamic size of the nanotube clusters, real time imaging (Fig 4 and supplementary information) reveals that single isolated nanotubes can also act as the primary units of the directed-assembly process leading to a percolation path.

\section{CONCLUSIONS}

Carbon structures of a wide range of geometries, sizes, concentrations and compositions form an electric-field induced conduction path in FC-40 due to the DEP force resulting from a nonuniform, externally applied, DC field. When the applied voltage exceeded their respective $V_{t}$, the suspended structures moved towards the electrodes, which $\mathrm{COMSOL}^{\circledR}$ simulations predict to be the regions of highest the field gradients. The model is also consistent with the experimentally observed field enhancement due to electrode "decoration" by the nanostructures. As predicted by the percolation theory, the measured $V_{t}$ values were found to be inversely proportional to the particle concentration. A direct proportionality between $V_{t}$ and the particle size indicated that the particle agglomerate model with simple hydrodynamic particle size measurements gives a qualitatively accurate description of the relationship between the two parameters. Notably, EGPs and MWCNTs exhibited lower $V_{t}$ and $C_{t}$ values compared to bulk graphite, and the $C_{t}$ values 
were found to exhibit a logarithmic relationship with the agglomerate size measured by dynamic light scattering.

\section{ACKNOWLEDGEMENT}

This work was supported by Savanah River National Lab under the U.S. DOE Contract DEAC09-96SR18500.

\section{REFERENCES:}

[1] Whitesides GM, Grzybowski B. Self-Assembly at All Scales. Science. 2002;295(5564):2418-21.

[2] Camazine S, Deneubourg J-L, Franks NR, Sneyd J, Theraulaz G, Bonabeau E. Self-Organization in Biological Systems: Princeton University Press; 2003.

[3] Mlot NJ, Tovey CA, Hu DL. Fire ants self-assemble into waterproof rafts to survive floods. Proceedings of the National Academy of Sciences. 2011;108(19):7669-73.

[4] Park WJ, Choi KJ, Kim MH, Koo BH, Lee J-L, Baik JM. Self-Assembled and Highly Selective Sensors Based on Air-Bridge-Structured Nanowire Junction Arrays. ACS Applied Materials \& Interfaces. 2013;5(15):6802-7.

[5] Saeedi E, Kim S, Parviz BA. Self-assembled crystalline semiconductor optoelectronics on glass and plastic. Journal of Micromechanics and Microengineering. 2008;18(7).

[6] Hynninen A-P, Thijssen JHJ, Vermolen ECM, Dijkstra M, van Blaaderen A. Self-assembly route for photonic crystals with a bandgap in the visible region. Nat Mater. 2007;6(3):202-5.

[7] Valkama S, Kosonen H, Ruokolainen J, Haatainen T, Torkkeli M, Serimaa R, et al. Self-assembled polymeric solid films with temperature-induced large and reversible photonic-bandgap switching. Nat Mater. 2004;3(12):872-6.

[8] Snow ES, Novak JP, Campbell PM, Park D. Random networks of carbon nanotubes as an electronic material. Applied Physics Letters. 2003;82(13):2145-7.

[9] Cao Q, Kim H-s, Pimparkar N, Kulkarni JP, Wang C, Shim M, et al. Medium-scale carbon nanotube thin-film integrated circuits on flexible plastic substrates. Nature. 2008;454(7203):495-500.

[10] Wu Z, Chen Z, Du X, Logan JM, Sippel J, Nikolou M, et al. Transparent, Conductive Carbon Nanotube Films. Science. 2004;305(5688):1273-6.

[11] Bradley K, Gabriel J-CP, Grüner G. Flexible Nanotube Electronics. Nano Letters. 2003;3(10):13535.

[12] Cao Q, Hur SH, Zhu ZT, Sun YG, Wang CJ, Meitl MA, et al. Highly Bendable, Transparent Thin-Film Transistors That Use Carbon-Nanotube-Based Conductors and Semiconductors with Elastomeric Dielectrics. Advanced Materials. 2006;18(3):304-9.

[13] Sun D-M, Timmermans MY, Kaskela A, Nasibulin AG, Kishimoto S, Mizutani T, et al. Mouldable all-carbon integrated circuits. Nat Commun. 2013;4.

[14] Stendahl JC, Rao MS, Guler MO, Stupp SI. Intermolecular forces in the self-assembly of peptide amphiphile nanofibers. Adv Funct Mater. 2006;16(4):499-508.

[15] Lumsdon SO, Kaler EW, Velev OD. Two-Dimensional Crystallization of Microspheres by a Coplanar AC Electric Field. Langmuir. 2004;20(6):2108-16.

[16] Martin JE, Snezhko A. Driving self-assembly and emergent dynamics in colloidal suspensions by time-dependent magnetic fields. Rep Prog Phys. 2013;76(12).

[17] Lee W, Amini H, Stone HA, Di Carlo D. Dynamic self-assembly and control of microfluidic particle crystals. P Natl Acad Sci USA. 2010;107(52):22413-8. 
[18] Yao SH, Dang ZM, Jiang MJ, Xu HP, Bai JB. Influence of aspect ratio of carbon nanotube on percolation threshold in ferroelectric polymer nanocomposite. Applied Physics Letters. 2007;91(21).

[19] Grzelczak M, Vermant J, Furst EM, Liz-Marzán LM. Directed Self-Assembly of Nanoparticles. ACS Nano. 2010;4(7):3591-605.

[20] Hermanson KD, Lumsdon SO, Williams JP, Kaler EW, Velev OD. Dielectrophoretic Assembly of Electrically Functional Microwires from Nanoparticle Suspensions. Science. 2001;294(5544):1082-6.

[21] Andrews R, Jacques D, Rao AM, Derbyshire F, Qian D, Fan X, et al. Continuous production of aligned carbon nanotubes: a step closer to commercial realization. Chem Phys Lett. 1999;303(5-6):46774.

[22] Liu YL, Chung JH, Liu WK, Ruoff RS. Dielectrophoretic assembly of nanowires. J Phys Chem B. 2006;110(29):14098-106.

[23] Xu Y, Liu Q, Zhu Y, Liu Y, Langrock A, Zachariah MR, et al. Uniform Nano-Sn/C Composite Anodes for Lithium Ion Batteries. Nano Letters. 2013;13(2):470-4. 